\title{
Towards a Rational Design of Laser-Coolable Molecules: Insights from Equation-of-Motion Coupled-Cluster Calculations
}

\author{
Maxim V. Ivanov ${ }^{a}$, Felix H. Bangerter ${ }^{a, b}$ and Anna I. Krylov ${ }^{a}$ \\ ${ }^{a}$ Department of Chemistry, University of Southern California, Los Angeles, California, USA \\ ${ }^{b}$ Ludwig Maximilian University Munich, Munich, Bavaria, Germany
}

Access to cold molecules is critical for quantum information science, design of new sensors, ultracold chemistry, and search of new phenomena. These applications depend on the ability to laser-cool molecules. Theory and qualitative models can play a central role in narrowing down the vast pool of potential candidates amenable to laser cooling. We report a systematic study of structural and optical properties of alkaline earth metal derivatives in the context of their applicability in laser cooling using equation-of-motion coupled-cluster methods. To rationalize and generalize the results from high-level electronic structure calculations, we develop an effective Hamiltonian model. The model explains the observed trends and suggests new principles for the design of laser-coolable molecules.

\section{INTRODUCTION}

The ability to bring atoms into the ultracold regime by means of laser cooling has opened new frontiers in physics and chemistry ${ }^{1,2}$. The access to cold polyatomic polar molecules holds even a greater promise in advancing such fundamental areas as quantum information science ${ }^{3,4}$, ultracold chemistry ${ }^{5-7}$, and precision measurements ${ }^{8}$, due to much richer electronic structure of molecules relative to atoms.

Laser cooling is achieved by a continuous scattering of a large number $\left(>10^{4}\right)$ of photons off the target, with each cycle of absorption and emission slowing down its translational motion, as illustrated in Figure 1. In contrast to atoms, where the spontaneous emission returns the excited atom to its original ground state, the emission in a molecule may populate numerous vibrational and rotational energy levels. In such a case, additional repump lasers must be used to bring the population back, in order to continue the photon cycling. It has been estimated that in order to bring at least $10 \%$ of molecules from $100 \mathrm{~m} / \mathrm{s}$ to rest by scattering 4,500 


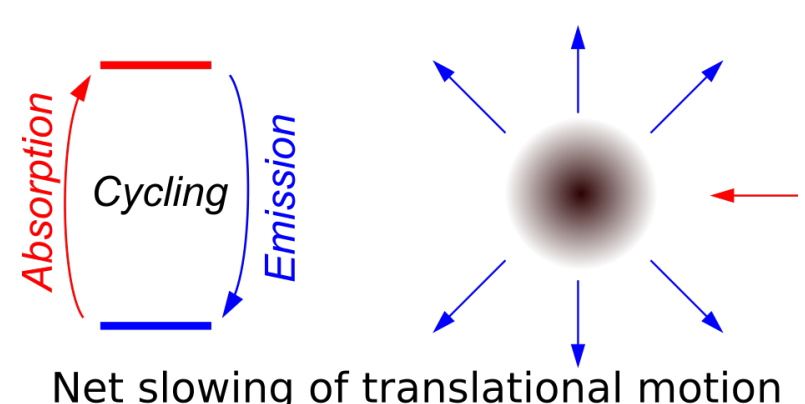

FIG. 1: Laser cooling is achieved by a large number of absorption and emission cycles (hence, cycling). An atom moving towards the laser absorbs the photon that slows it down via spontaneous emission at random direction with no net change in the momentum over many absorption-emission cycles.

photons, $99.95 \%$ of the emission has to be recovered by the lasers ${ }^{9}$. Therefore, the key feature of a prospective laser-coolable molecule is diagonal Franck-Condon factors (FCFs), implying that the shape of the potential energy surface (PES) of the two electronic states involved in the cycling should have similar shapes.

One class of species with nearly parallel ground and electronically excited PES are molecules in which a single unpaired electron is localized at the cycling (usually metal) center and the electronic excitations resemble atomic transitions, as illustrated in the left panel of Fig. 2. This relatively simple atom-like electronic structure leads to minimal structural differences between the ground and excited states $^{9}$. Consequently, because the spontaneous emission in these molecules is confined only to a few vibronic branches, laser cooling is possible with only a few repump lasers (Fig. 2, right panel).

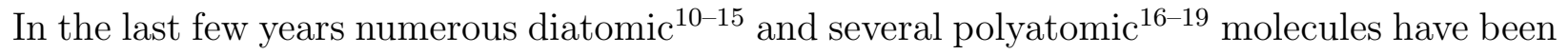
computationally studied as candidates for laser cooling. Yet, only a few have been laser cooled to (sub-)millikelvin temperatures, as summarized in Table I. Among these are the free radical derivatives of alkaline earth metals, a rare earth metal and a lanthanide ${ }^{20-27}$.

As demonstrated by a recent breakthrough by Doyle and co-workers ${ }^{28}$, who laser-cooled $\mathrm{SrOH}$ to submilikelvin temperature, laser cooling of triatomic and possibly even larger molecules is possible ${ }^{29,30}$, further expanding the chemical space of the candidate molecules. In this context, the role of reliable electronic structure methods that could accurately predict structural and optical properties of novel candidate molecules becomes increasingly important.

The majority of the systems proposed in the literature have been theoretically studied using complete active space self-consistent field (CASSCF) and multireference configuration inter- 


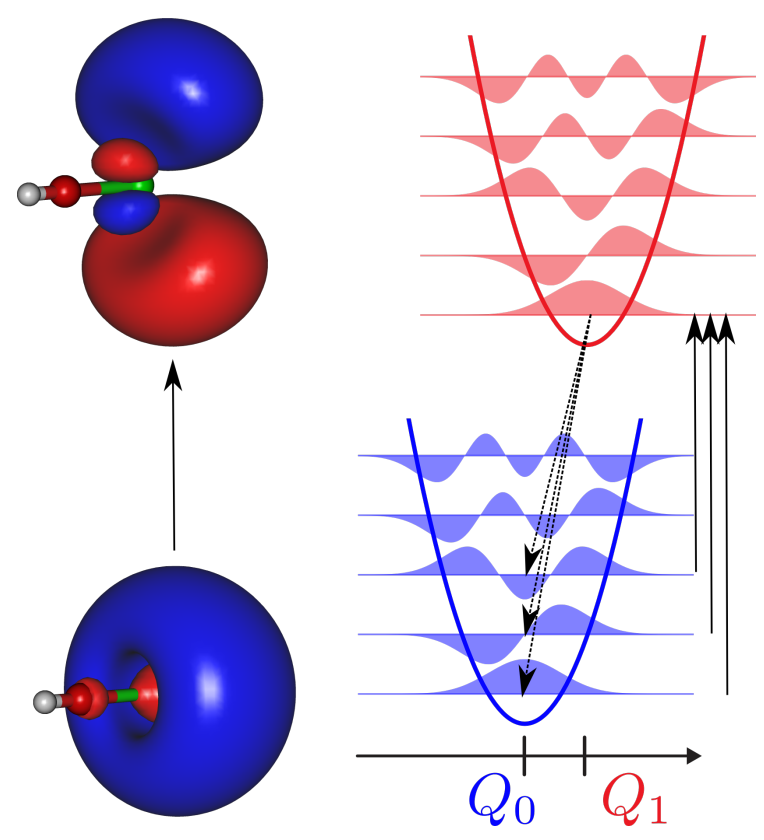

FIG. 2: Left: Natural transition orbitals for the $X^{2} \Sigma \rightarrow A^{2} \Pi$ transition in SrOH. Right:

Cartoon representing decay paths (dashed lines) and laser transitions (solid lines). In laser-coolable molecules, the spontaneous emission must be confined only to a few vibronic branches with a dominant decay to the ground vibrational level $(\mathrm{FCF} \gg 0.9)$.

TABLE I: Molecules that have been laser cooled, the electronic transitions used for cycling and the lowest temperature $(\mathrm{T})$ that has been achieved. Energies of all transitions lie in $1.8-2.3 \mathrm{eV}$ range.

\begin{tabular}{|l|l|l|l|}
\hline Molecule & Transition & $\mathrm{T}$ & Source \\
\hline $\mathrm{SrF}$ & $X^{2} \Sigma^{+}-A^{2} \Pi_{1 / 2}$ & $2.5 \mathrm{mK}$ & Refs. 20,21 \\
\hline $\mathrm{YO}$ & $X^{2} \Sigma^{+}-A^{2} \Pi_{1 / 2}$ & $2 \mathrm{mK}$ & Refs. 22,23 \\
\hline \multirow{2}{*}{$\mathrm{CaF}$} & $X^{2} \Sigma^{+}-A^{2} \Pi_{1 / 2}$ & $60 \mu \mathrm{K}$ & Refs. 24,25 \\
& $X^{2} \Sigma^{+}-B^{2} \Sigma^{+}$ & $50 \mu \mathrm{K}$ & Ref. 26 \\
\hline $\mathrm{YbF}$ & $X^{2} \Sigma^{+}-A^{2} \Pi_{1 / 2}$ & $100 \mu \mathrm{K}$ & Ref. 27 \\
\hline \multirow{2}{*}{$\mathrm{SrOH}$} & $X^{2} \Sigma^{+}-A^{2} \Pi_{1 / 2}$ & $2 \mathrm{mK}$ & Ref. 28 \\
& $X^{2} \Sigma^{+}-B^{2} \Sigma^{+}$ & $750 \mu \mathrm{K}$ & Ref. 28 \\
\hline
\end{tabular}

action (MRCI) methods. By using these tools, accurate potential energy curves of several diatomic molecules were constructed in order to compute FCFs. Although capable of producing highly accurate results, these methods quickly become prohibitively expensive due to their steep computational scaling. Even more importantly, their application involves laborious and system-specific process of selecting the active space and determining the protocols for state averaging. These system-specific parameters and the violation of size extensivity prevent efficient explorations of the vast chemical space in search for the laser-coolable molecules. Thus, for an extensive computational screening of a large number of polyatomic molecules it is desirable 
to employ more robust and black-box methods. Therefore, here we employ equation-of-motion coupled-cluster (EOM-CC) approach, a versatile electronic-structure tool capable of describing a variety of multiconfigurational wave functions within the single-reference formalism ${ }^{31-33}$.

In addition to a reliable and accurate computational method that can deliver high-quality numeric results, it is important to develop an intuitive physical model that can guide the search of new candidate systems for their further computational and experimental verification. With a goal to develop such molecular design principles, we carried out a systematic study of structural and optical properties of alkaline earth metal derivatives using the EOM-CC methods. In particular, we investigate the roles of specific alkaline earth metals and attached ligands on the overall properties a molecule. Here we report the results of the calculations of alkaline earth metal derivatives with the general chemical formula $\mathrm{MR}$, where $\mathrm{M}=\mathrm{Ca}, \mathrm{Sr}, \mathrm{Ba}$ is attached to ligands with a varied electron-withdrawing strength (i.e., $\mathrm{R}=\mathrm{H}, \mathrm{CCH}, \mathrm{OH}, \mathrm{F}, \mathrm{NCO}, \mathrm{NC}, \mathrm{OBO}$ ). To explain the trends in computed excitation energies, oscillator strengths, and FCFs, we developed a simple qualitative model based on an effective Hamiltonian. The model is constructed

in the spirit of the ligand field approach ${ }^{34-36}$ and is based on the Hamiltonian of hydrogen-like atom augmented by a point-charge perturbing potential, which accounts for the long-range interactions of the unpaired electron with the ligand. The short-range core-penetrating effects ${ }^{37}$ are included via quantum defect theory, which is often employed for describing Rydberg states in atoms ${ }^{38}$, diatomic $^{39-42}$ and polyatomic ${ }^{43,44}$ molecules.

By combining the results of the high-level electronic structure calculations with the proposed model, we explain the observed trends and conclude that the molecules with the most favorable FCFs are expected to have the quantum defect that is the lowest in each alkaline earth metal series and the ligand with the largest electron-withdrawing strength. These findings provide new insights into the rational design principles of novel candidates for laser cooling.

\section{THEORETICAL METHODS AND COMPUTATIONAL DETAILS}

The EOM-CC theory provides an efficient and robust framework for accurate description of closed- and open-shell species in the ground and electronically excited states ${ }^{31-33,45}$. As a multi-state method, EOM-CC is an excellent platform for computational spectroscopy. EOM$\mathrm{CC}$ treats dynamical and non-dynamical correlation in the same computational scheme, which results in a balanced description of states of different character. It is rigorously size-intensive, 
which enables unambiguous comparisons between series of molecules.

The species studied here contain an alkaline earth metal $(\mathrm{M}=\mathrm{Ca}, \mathrm{Sr}, \mathrm{Ba})$ attached to a substituent group R, giving rise to one unpaired electron. As illustrated in Figure 3, such doublet states are best described by EOM-EA-CC (EOM-CC for electron-attached states) using a closed-shell cationic state as the reference. Here we employ EOM-CC with single and double substitutions (EOM-EA-CCSD) in which the reference state is described by CCSD and the excitation operators $R$ are of the 1-particle and 2-particle-1-hole types.

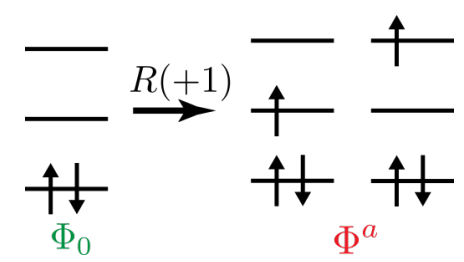

FIG. 3: EOM-EA ansatz allows access to open-shell doublet states. The target $\Psi(N)$ states are reached by applying the excitation operator $R(+1)$, which adds one electron the closed-shell reference $\Psi_{0}(N-1)$ with $N-1$ electrons: $\Psi(N)=R(+1) \Psi_{0}(N-1)$.

Just as in other areas of chemistry and physics, molecular orbital (MO) theory is instrumental in the rational design of novel molecules and materials ${ }^{46,47}$. It provides key concepts for characterizing electronic states and transitions between them. Despite its origins in the mean-field Hartree-Fock description of pseudo non-interacting electrons, the MO theory can be extended to correlated many-electron wave functions via generalized one-electron quantities such as Dyson ${ }^{48-50}$ and natural transition orbitals (NTOs) $)^{51-57}$.

Dyson orbital is defined as the overlap between $N$ and $N$-1-electron wavefunctions ${ }^{48,49,58}$ :

$$
\varphi_{I F}^{d}=\sqrt{N} \int \Phi_{F}^{N}(1, \ldots, n) \Phi_{I}^{N-1}(2, \ldots, n) d 2 \ldots d n
$$

where $I$ and $F$ denote the two many-body states (e.g., of the neutral and of the cation). We use Dyson orbitals between the cationic reference state and the doublet neutral states of the MR species to characterize the spatial distribution of the unpaired electron.

NTOs allow one to describe electronic transitions between many-body states in terms of the minimal number of hole-electron excitations ${ }^{51-57}$. We use NTOs to quantify the locality of electronic transitions. The difference between two states in terms of one-electron excitations is 
given by one-particle transition density matrix

$$
\gamma_{p q}^{F I}=\left\langle\Phi_{F}\left|\hat{p}^{\dagger} \hat{q}\right| \Phi_{I}\right\rangle
$$

which can be used to define exciton wavefunction

$$
\Psi_{e x c}\left(r_{h}, r_{e}\right)=\sum_{p q} \gamma_{p q}^{F I} \varphi_{p}\left(r_{h}\right) \varphi_{q}\left(r_{e}\right)
$$

which describes the redistribution of electron density upon excitation. One-particle density matrix (or exciton wave function) contain contain all information needed to compute oneelectron interstate properties, such as transition dipole moment matrix elements. By using singular value decomposition of $\gamma_{p q}$, the exciton wavefunction can be written as

$$
\Psi_{e x c}\left(r_{h}, r_{e}\right)=\sum_{K} \sigma_{K} \psi_{K}^{h}\left(r_{h}\right) \psi_{K}^{e}\left(r_{e}\right)
$$

where $\sigma_{K}$ are singular values, $\psi_{K}^{h}\left(r_{h}\right)$ are hole orbitals, and $\psi_{K}^{e}\left(r_{e}\right)$ are particle orbitals. Usually, only a few singular values are significant. Thus, NTOs allow one to express the difference between two correlated many-body wave functions in terms of pairs of hole and particle orbitals. NTOs are directly related to the observables, because transition properties can be expressed as matrix elements between the hole and particle pairs:

$$
\mu^{I F}=\left\langle\Psi^{F}|\mu| \Psi^{I}\right\rangle=\operatorname{Tr}\left[\gamma^{F I} \mu\right]=\sum_{K} \sigma_{K}\left\langle\psi_{K}^{h}|\mu| \psi_{K}^{e}\right\rangle
$$

\section{A. Computational Details}

All electronic structure calculations were performed using the $Q$-Chem package ${ }^{59,60}$. Prior studies have shown that in order to properly describe the electronic structure of alkaline earth metal containing molecules, it is important to include core-valence correlation ${ }^{61,62}$. Therefore, we employed the aug-cc-pwCVTZ-PP basis set ${ }^{63}$ with small-core pseudo-potentials ${ }^{64,65}$ to treat $\mathrm{Ca}$, Sr and $\mathrm{Ba}$ and aug-cc-pVTZ ${ }^{66}$ to treat remaining atoms. All electrons except for the core electrons of the ligand were correlated. Atomic charges were computed using the Mulliken $^{67}$ and natural ${ }^{68}$ population analyses. The NTO analysis was carried out using libwfa library $^{55}$. Calculations of FCFs were carried out within the double-harmonic approximation 
using ezSpectrum ${ }^{69}$.

\section{RESULTS}

\section{A. Electronic Structure of Alkaline Earth Metal Derivatives}

Various monovalent derivatives of alkaline earth metals have been investigated using highresolution spectroscopy and $a b$ initio calculations ${ }^{70-74}$. Among previously investigated systems, M-F and M-OH species have been studied in the greatest detail. Therefore, we begin by examining the electronic structure of $\mathrm{MF}$ and $\mathrm{MOH}(\mathrm{M}=\mathrm{Ca}$, Sr and $\mathrm{Ba})$ using EOM-EACCSD, and wavefunction analysis tools and compare our results with the available experimental data.

Monovalent alkaline earth metals derivatives $\mathrm{MR}$ can be described ${ }^{75}$ as ionic compounds in which one of the two valence $s$ electrons of the metal is transferred to the ligand: $M^{+} L^{-}$. For example, in $\mathrm{SrOH}$, the calculations yield Mulliken's charge +0.77 on the strontium atom, which illustrates strongly ionic bond in these species.

The interaction between $\mathrm{M}^{+}$and $\mathrm{R}^{-}$is manifested in the orbital hybridization on the metal atom, which minimizes the repulsion between the unpaired electron and a negatively charged ligand. Indeed, in the ground $X^{2} \Sigma^{+}$state of $\mathrm{SrOH}$, the Dyson orbital shows that the unpaired electron is localized at the metal and occupies the $5 s \sigma$ orbital mixed with the $5 p \sigma$ orbital (Figure 4). The electron is also exclusively localized on the metal in the $A^{2} \Pi$ and $B^{2} \Sigma^{+}$states, occupying the $5 p \pi-4 d \pi$ and $5 p \sigma-4 d \sigma$ hybridized orbitals, respectively. The Dyson orbital for the third excited (dark) $A^{\prime 2} \Delta$ state is of a pure $d$ character.

The transition from the ground state to any of the three lowest excited states can be represented by a single pair of NTOs. The calculations show that for all three transitions the hole NTOs are nearly identical to the Dyson orbital of the $X^{2} \Sigma^{+}$state, whereas their particle NTOs are similar to the Dyson orbitals of the respective excited state (Figure 5).

Changing the metal from $\mathrm{Sr}$ to either $\mathrm{Ca}$ or $\mathrm{Ba}$ does not change the character of Dyson orbitals and NTOs (Figure S1 in SI), yet the excitation energies and transition properties depend strongly on the metal. Changing the metal from Ca to $\mathrm{Sr}$ to Ba, the excitation energies decrease, whereas the transition dipole moments increase (Table II).

Optical properties of MR can also be tuned by varying the electron-withdrawing strength 
of the ligand. For example, change of the ligand in $\mathrm{SrR}$ from $\mathrm{OH}$ to $\mathrm{F}$ withdraws more of the electron density from the metal, as indicated by the increase of the Mulliken's charge on Sr to +0.83 . This, in turn, leads to a systematic increase of the excitation energies of the $A^{2} \Pi$ and $B^{2} \Sigma^{+}$states by $0.04-0.12 \mathrm{eV}$ and a slight decrease of the excitation energies of the $A^{\prime 2} \Delta$ state by $0.03-0.06 \mathrm{eV}$.

In $\mathrm{BaOH}$ and $\mathrm{BaF}$, the order of the excited states differs from that in the Ca and Sr derivatives: the $A^{\prime 2} \Delta$ states are stabilized and become near-degenerate with the $A^{2} \Pi$ states (Table II). In the context of laser cooling, the presence of this low-lying electronic state may create problems due to the additional population leakage ${ }^{74}$.

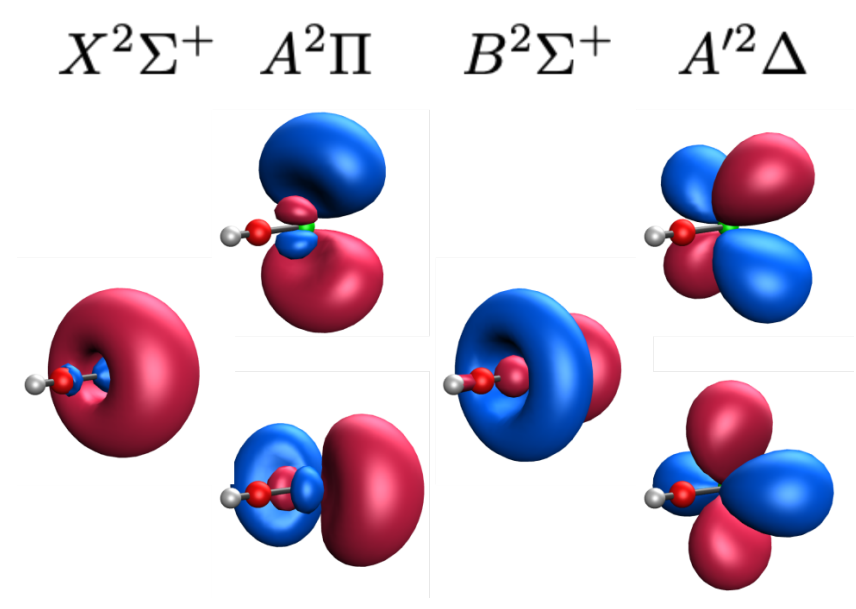

FIG. 4: Dyson orbitals (isovalue $=0.020$ ) of four lowest electronic states in $\mathrm{SrOH}$.

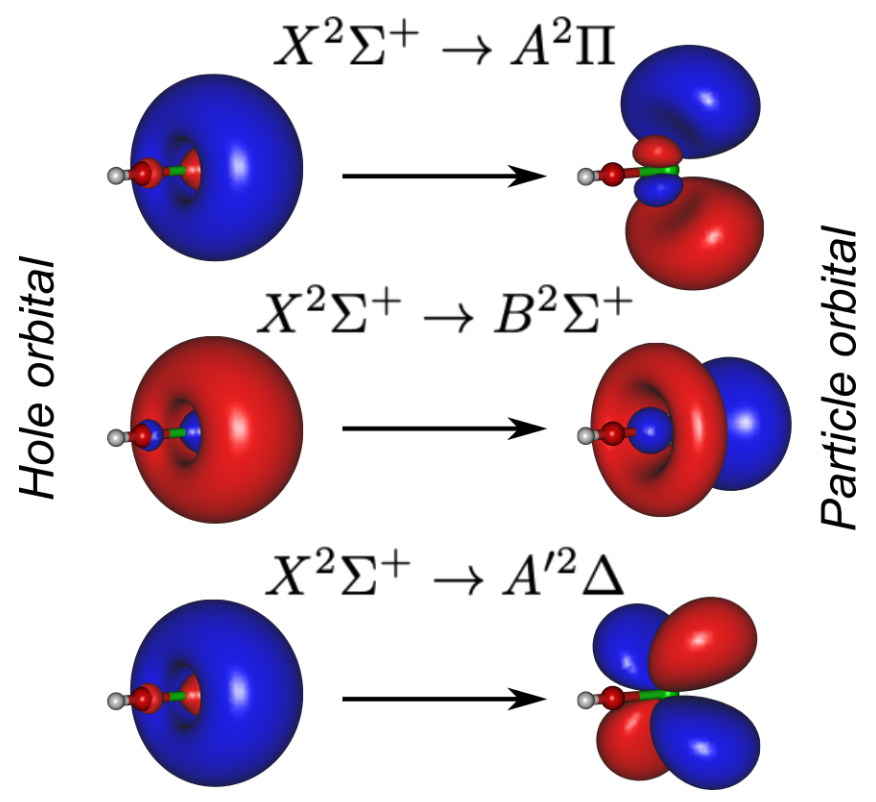

FIG. 5: NTOs (isovalue $=0.025$ ) for the three lowest-energy transitions in SrOH. 
TABLE II: Vertical excitation energies $\left(E_{e x}\right)$, oscillator strength $\left(f_{\text {osc }}\right)$, transition dipole moment $\left(\mu^{I F}\right)$, excitation-induced change in the M-X bond length $(\Delta R)$ of $\mathrm{MR}(\mathrm{M}=\mathrm{Ca}, \mathrm{Sr}$, $\mathrm{Ba}$ and $\mathrm{R}=\mathrm{OH}, \mathrm{F})$.

\begin{tabular}{cccccc}
\hline Molecule State & $E_{\text {ex }}, \mathrm{eV}$ & $f_{\text {osc }}$ & $\mu^{I F}, \mathrm{au}$ & $\Delta R, \AA$ \\
\hline \multirow{6}{*}{$\mathrm{CaOH}$} & $A^{2} \Pi$ & 2.03 & 0.261 & 2.287 & -0.026 \\
& $B^{2} \Sigma^{+}$ & 2.34 & 0.197 & 1.854 & -0.024 \\
& $A^{\prime 2} \Delta$ & 2.98 & 0.000 & 0.000 & - \\
\hline \multirow{3}{*}{$\mathrm{SrOH}$} & $A^{2} \Pi$ & 1.83 & 0.275 & 2.473 & -0.024 \\
& $B^{2} \Sigma^{+}$ & 2.07 & 0.205 & 2.011 & -0.018 \\
& $A^{\prime 2} \Delta$ & 2.50 & 0.000 & 0.000 & - \\
\hline \multirow{3}{*}{$\mathrm{BaOH}$} & $A^{2} \Pi$ & 1.47 & 0.240 & 2.580 & 0.030 \\
& $A^{\prime 2} \Delta$ & 1.52 & 0.000 & 0.000 & - \\
& $B^{2} \Sigma^{+}$ & 1.64 & 0.179 & 2.109 & 0.022 \\
\hline \multirow{3}{*}{$\mathrm{CaF}$} & $A^{2} \Pi$ & 2.10 & 0.265 & 2.268 & -0.019 \\
& $B^{2} \Sigma^{+}$ & 2.44 & 0.196 & 1.811 & -0.005 \\
& $A^{\prime 2} \Delta$ & 2.94 & 0.000 & 0.000 & - \\
\hline \multirow{3}{*}{$\mathrm{SrF}$} & $A^{2} \Pi$ & 1.90 & 0.277 & 2.437 & -0.017 \\
& $B^{2} \Sigma^{+}$ & 2.18 & 0.205 & 1.959 & 0.000 \\
& $A^{\prime 2} \Delta$ & 2.47 & 0.000 & 0.000 & - \\
\hline \multirow{3}{*}{$\mathrm{BaF}$} & $A^{\prime 2} \Delta$ & 1.46 & 0.000 & 0.000 & - \\
& $A^{2} \Pi$ & 1.51 & 0.221 & 2.443 & 0.014 \\
& $B^{2} \Sigma^{+}$ & 1.76 & 0.182 & 2.050 & 0.042 \\
\hline
\end{tabular}


Continuing with $\mathrm{SrOH}$ as a representative molecule, in the ground electronic state it is a linear molecule that remains linear upon electronic excitation. Relative to the ground state, the Sr-O bond length is shortened by $0.024 \AA$ in the $A^{2} \Pi$ state and by $0.018 \AA$ in the $B^{2} \Sigma^{+}$ state (Table II). Change in the $\mathrm{O}-\mathrm{H}$ bond length is negligible, within $0.001 \AA$. As a triatomic linear molecule, SrOH has a doubly degenerate bending mode and two stretching Sr-O and $\mathrm{O}-\mathrm{H}$ modes. Therefore, spontaneous emission is expected to be confined mainly to the Sr-O stretching mode due to a non-negligible displacement in Sr-O bond. A decay into the bending mode could be facilitated by large anharmonic effects and/or by a large change in the frequency of the bending mode.

FCFs calculations within double-harmonic approximation show that 0.0610 of decay from $A^{2} \Pi$ is confined to the Sr-O stretching mode, 0.0006 of decay is confined to the bending mode due to the differences in frequencies, and the remaining 0.9383 fraction of the total population goes back to the ground state (Table III). FCFs for the $B^{2} \Sigma^{+}$state are somewhat more diagonal due to a smaller change in the Sr-O bond length. Because of the similar bond length changes in $\mathrm{CaOH}$, the respective FCFs are also quite similar to those in $\mathrm{SrOH}$ (Tables II and III).

Changing the substituent from $\mathrm{OH}$ to $\mathrm{F}$ decreases the magnitude of the bond length change (Table II), resulting in more diagonal FCFs in MF (compare Tables III and IV). Interestingly, in both $\mathrm{BaOH}$ and $\mathrm{BaF}$ the bond between the metal and directly attached atom of the ligand (abbreviated as M-X) undergoes elongation rather than contraction upon the excitation to the $A^{2} \Pi$ and $B^{2} \Sigma^{+}$states (Table II). The magnitude of the change is larger than in $\mathrm{CaOH}$ and SrOH, resulting in less diagonal FCFs (Table III).

Our analysis shows that the computed structural and optical parameters agree well with the available experimental data: the adiabatic excitation energies deviate from the experimental $T_{e}$ values by $0.1 \mathrm{eV}$ or less (Table $\mathrm{S} 1$ in $\mathrm{SI}$ ), the equilibrium bond lengths differ from the experimental $r_{e}$ values by 0.001-0.032 $\AA$, the computed bond length changes agree with the experimental values within 0.003-0.006 $\AA$ (Table S2 in SI), and, consequently, the relative errors of the computed FCFs for 0-0 transitions are low, around 2-3\% (Table III).

In light of the observed appreciable dependence of the structural and optical properties on the electron-withdrawing strength of the ligand, it is important to understand how these properties change when the amount of charge on the cycling center is varied systematically. Towards this goal, we examine electronic structure of MR with varied ligands, starting from $\mathrm{R}$ $=\mathrm{H}$ and up to superhalogen $\mathrm{OBO}$ with the largest known electron affinity (Table S3 in SI) ${ }^{76,77}$. 
TABLE III: FCFs for the decay transitions from the $A^{2} \Pi$ and $B^{2} \Sigma^{+}$states to $X^{2} \Sigma^{+}$state in $\mathrm{MOH}$ calculated with EOM-EA-CCSD and the corresponding experimental data for $\mathrm{CaOH}$ (Ref. 78) and $\mathrm{SrOH}$ (Ref. 79). The experimental values of the normal mode frequencies have been used in the calculations of FCFs for $\mathrm{CaOH}$ and $\mathrm{SrOH} . \omega_{1}$ is the M-O stretching mode, $\omega_{2}$ is the M-O-H bending mode, and $\omega_{3}$ is the $\mathrm{O}-\mathrm{H}$ stretching mode.

\begin{tabular}{c|c|c|c|c|c}
\hline \multirow{2}{*}{ Transition } & EOM-EA-CCSD & \multicolumn{3}{c}{ Experiment } \\
\cline { 2 - 6 } & $A^{2} \Pi$ & $B^{2} \Sigma$ & $A^{2} \Pi_{1 / 2}$ & $A^{2} \Pi_{3 / 2}$ & $B^{2} \Sigma$ \\
\hline \multicolumn{7}{c}{$\mathrm{CaOH}$} \\
\hline $0_{0}^{0}$ & 0.9287 & 0.9371 & $0.957 \pm 0.002$ & $0.959 \pm 0.005$ & $0.975 \pm 0.001$ \\
$1_{1}^{0}$ & 0.0696 & 0.0615 & $0.043 \pm 0.002$ & $0.041 \pm 0.005$ & $0.022 \pm 0.001$ \\
$1_{2}^{0}$ & 0.0015 & 0.0011 & - & - & - \\
$2_{1}^{0}$ & 0.0000 & 0.0000 & - & - & $0.003 \pm 0.001$ \\
$2_{2}^{0}$ & 0.0002 & 0.0002 & $3_{-2}^{+1} \times 10^{-3}$ & - & - \\
\hline \multicolumn{5}{c}{$\mathrm{SrOH}$} & - \\
\hline $0_{0}^{0}$ & 0.9383 & 0.9605 & $0.957 \pm 0.003$ & $0.959 \pm 0.003$ & $0.977 \pm 0.002$ \\
$1_{1}^{0}$ & 0.0599 & 0.0368 & $0.043 \pm 0.002$ & $0.041 \pm 0.004$ & $0.023 \pm 0.003$ \\
$1_{2}^{0}$ & 0.0011 & 0.0004 & $<0.005$ & $<0.007$ & $<0.005$ \\
$2_{1}^{0}$ & 0.0000 & 0.0000 & - & - & - \\
$2_{2}^{0}$ & 0.0006 & 0.0022 & $<0.005$ & $<0.007$ & $<0.005$ \\
\hline \multicolumn{7}{c}{$\mathrm{BaOH}$} & - & - \\
\hline $0_{0}^{0}$ & 0.8716 & 0.9224 & - & - & - \\
$1_{1}^{0}$ & 0.1197 & 0.0485 & - & - & - \\
$1_{2}^{0}$ & 0.0082 & 0.0175 & - & - & - \\
$2_{1}^{0}$ & 0.0000 & 0.0000 & - & - & - \\
$2_{2}^{0}$ & 0.0000 & 0.0000 & - &
\end{tabular}

TABLE IV: FCFs for the decay transitions from the $A^{2} \Pi$ and $B^{2} \Sigma^{+}$states to $X^{2} \Sigma^{+}$in MF molecules calculated with EOM-EA-CCSD.

\begin{tabular}{c|cc|cc|cc}
\hline \multirow{2}{*}{ Transition } & \multicolumn{2}{|c}{$\mathrm{CaF}$} & \multicolumn{2}{c}{$\mathrm{SrF}$} & \multicolumn{2}{c}{$\mathrm{BaF}$} \\
\cline { 2 - 7 } & $A^{2} \Pi$ & $B^{2} \Sigma^{+}$ & $A^{2} \Pi$ & $B^{2} \Sigma^{+}$ & $A^{2} \Pi$ & $B^{2} \Sigma$ \\
\hline $0_{0}^{0}$ & 0.9609 & 0.9973 & 0.9645 & 0.9996 & 0.9799 & 0.8153 \\
$1_{1}^{0}$ & 0.0382 & 0.0027 & 0.0355 & 0.0004 & 0.0199 & 0.1738 \\
$1_{2}^{0}$ & 0.0008 & 0.0000 & 0.0000 & 0.0000 & 0.0002 & 0.0108 \\
\hline
\end{tabular}




\section{B. The Effect of A Substituent on Optical Properties of the MR molecules}

In order to probe how optical properties of alkaline earth metal derivatives vary with the electron-withdrawing strength of the ligand, we carried out EOM-EA-CCSD calculations of the $\mathrm{MR}$ series with $\mathrm{M}=\mathrm{Ca}, \mathrm{Sr}, \mathrm{Ba}$ and $\mathrm{R}=\mathrm{H}, \mathrm{CCH}, \mathrm{OH}, \mathrm{F}, \mathrm{NCO}, \mathrm{NC}, \mathrm{OBO}$. The results are presented graphically in Figs. 6-7; the numerical data are given in Tables S4 and S5 in SI.

Overall, excitation energies increase when the electron-withdrawing strength of the ligand increases. For all transitions (except $X^{2} \Sigma^{+} \rightarrow A^{2} \Pi$ in CaR and SrR series) the maximum excitation energies correspond to $\mathrm{MOH}$ and MF (Figure 6). At the same time, changing the metal from $\mathrm{Ca}$ to $\mathrm{Sr}$ to $\mathrm{Ba}$ leads to a systematic decrease in excitation energies. In Ba derivatives, the $A^{\prime 2} \Delta$ state is stabilized and becomes near-degenerate with the $A^{2} \Pi$ state.

Similarly to the trend in excitation energies, oscillator strengths increase with an increasing electron-withdrawing strength, with the largest values observed in MOH and MF (Figure S2 in $\mathrm{SI})$. In Ba derivatives, oscillator strengths are generally lower than in the corresponding CaR and SrR molecules, with $\mathrm{BaF}$ and $\mathrm{BaOH}$ showing the largest deviation from the overall trend.

Molecules remain linear in the excited states with the geometry changes corresponding mainly to the contraction/elongation of the M-X bond. The excitation-induced M-X bond length contraction in $\mathrm{CaR}$ and $\mathrm{SrR}$ are the largest for $\mathrm{MH}$ and decrease in magnitude as the electron-withdrawing strength increases (Figure 7). In contrast, in BaR the M-X bonds elongate and the magnitudes of the change are comparable for molecules with the smallest (i.e., $\mathrm{BaH}$ ) and the largest (i.e., BaNC, BaOBO) electron-withdrawing strength of the ligand. Despite the overall trend, in all three series of alkaline earth metal derivatives, the molecules with the ligands of moderate electron-withdrawing strength (i.e., $\mathrm{MF}$ and $\mathrm{MOH}$ ) exhibit the bond length changes that are among the smallest in each series.

The electron-withdrawing strength of the ligand affects the strength of the ionic bond between the metal and the ligand. As the electron-withdrawing strength increases, the strength of the bond and, thereby, the partial charge of metal increases. Thus, in order to achieve a qualitative understanding of the trends observed in the calculations, below we develop a model describing the energies of the valence electronic states as a function of the partial charge of the metal. The model exploits perturbation theory starting from the Hamiltonian of a hydrogen-like atom with charge $Z$ :

$$
H^{(0)}=-\frac{1}{2} \frac{d^{2}}{d r^{2}}+\frac{l^{2}}{2 r^{2}}-\frac{Z}{r}
$$




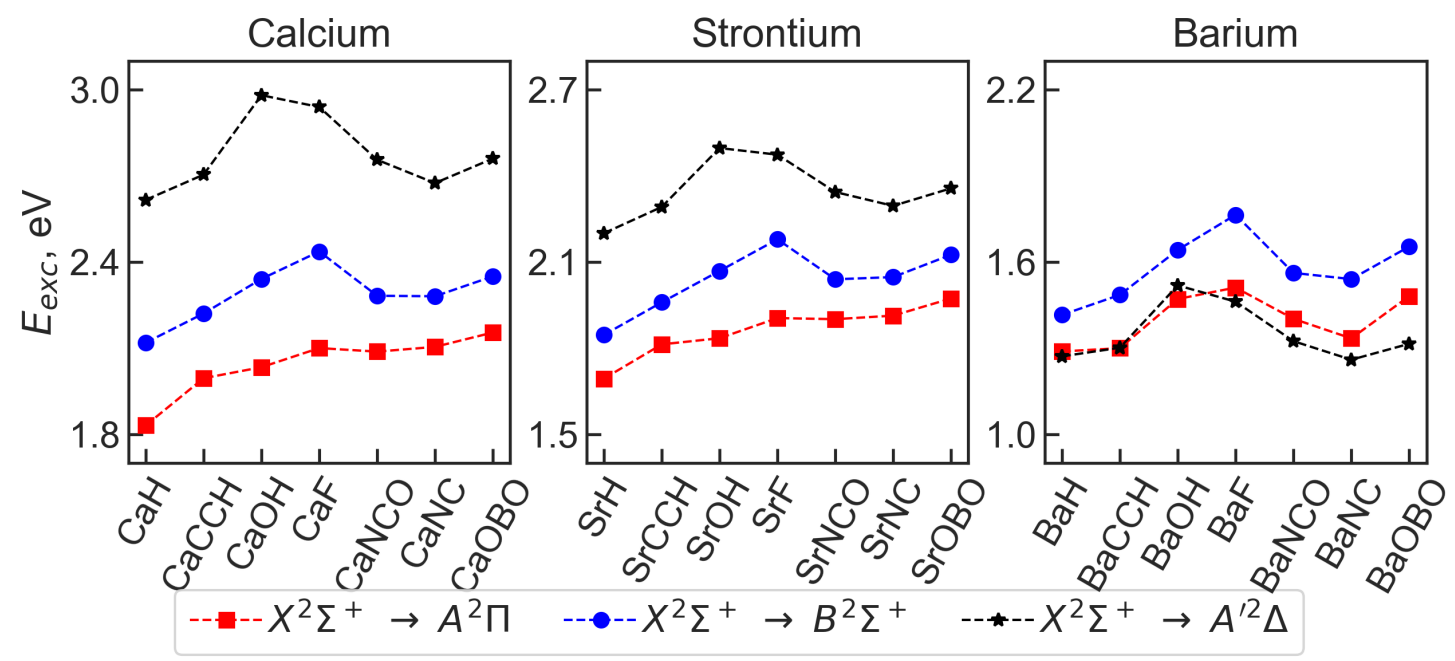

FIG. 6: Excitation energies of the three lowest-energy transitions (as indicated) in the MR molecules computed with EOM-EA-CCSD.
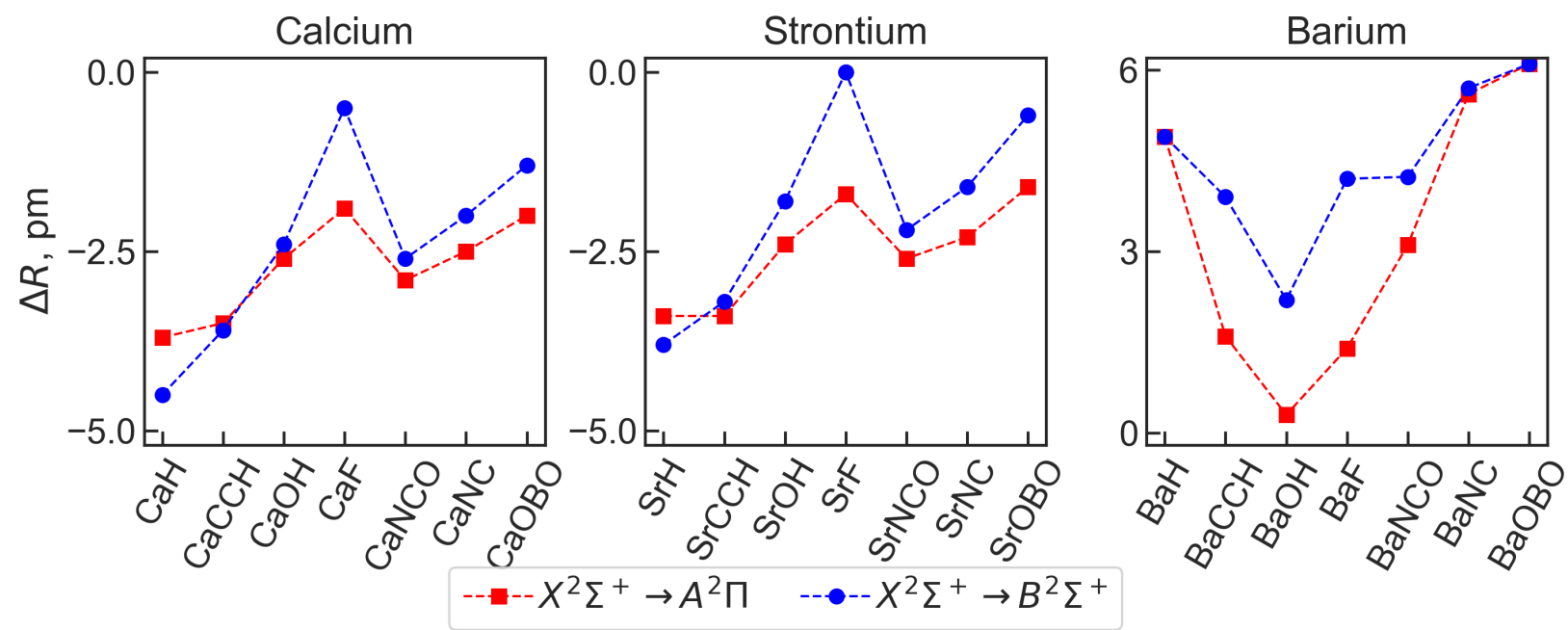

FIG. 7: Changes in the M-X bond length upon excitation to two lowest-energy excited states (as indicated) in the MR molecules computed with EOM-EA-CCSD.

where $\boldsymbol{l}$ is the angular momentum operator. The zero-order energies and wavefunctions are

$$
\begin{gathered}
E_{n}^{(0)}=-\frac{Z^{2}}{2 n^{2}}, \\
\psi_{n l m}^{(0)}=R_{n l}(r) Y_{l m}(\theta, \varphi),
\end{gathered}
$$

where $n$ is the principle quantum number, $l$ is the angular momentum quantum number, $m$ is the projection of the angular momentum on the $z$-axis, $Y_{l m}$ are spherical harmonics, $\varphi$ is polar angle, and $\theta$ is azimuthal angle. The effect of the ligand is described as a perturbation by the 
Coulomb potential of point charge $Z^{\prime}$ displaced by distance $d$ from the metal (Figure 8):

$$
V=-\frac{Z^{\prime}}{|\mathbf{r}-\mathbf{d}|}
$$

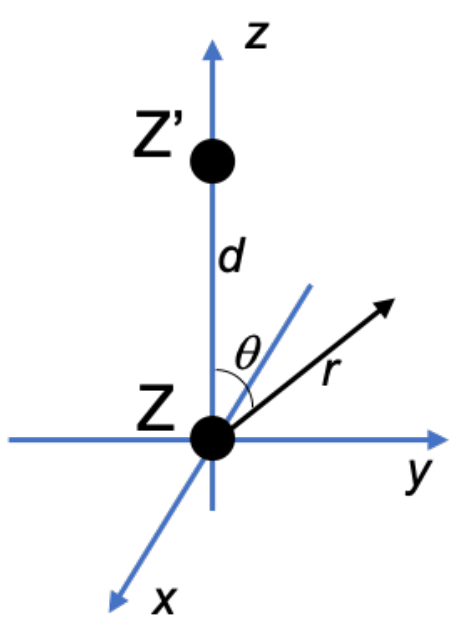

FIG. 8: The effect of the ligand is described by a point charge displaced by distance $d$ from the metal.

The Coulomb potential in Eq. (9) can be expanded in terms of spherical harmonics:

$$
V=-Z^{\prime} \sum_{l=0}^{\infty} \sum_{m=-l}^{l} \frac{4 \pi}{2 l+1} \frac{r_{<}^{l}}{r_{>}^{l+1}} Y_{l m}(\hat{\mathbf{r}}) Y_{l m}(\hat{\mathbf{d}})
$$

where $\hat{\mathbf{r}}=\mathbf{r} / r$ and $\hat{\mathbf{d}}=\mathbf{d} / r$ are unit vectors defined by $\varphi$ and $\theta, r_{<}$is the smaller and $r_{>}$is the larger of $r$ and $d$.

Here, we assume that $r>d$ and truncate the expansion after the dipole term. Using the definition of the total charge $Q=Z+Z^{\prime}$ and dipole moment $\mu=Z^{\prime} d$ of the molecular core, the perturbation can be rewritten as:

$$
V=\frac{Z}{r}-\frac{Q}{r}+\frac{\mu \cos \theta}{r^{2}}
$$

where the first term accounts for the repulsive interaction between the Rydberg electron and the negatively charged ligand, while the second and third term represent long-range interaction with the first two leading multipoles of the molecular core. The first-order correction to the 
energy has contribution only from the first two terms:

$$
E_{n}^{(1)}=\left\langle n l m|V| n l^{\prime} m\right\rangle=\frac{Z}{n^{2}}-\frac{Q}{n^{2}}
$$

The contribution from the third (dipole) term is zero as the angular factor in matrix element $\left\langle n l m\left|\cos \theta r^{-2}\right| n l^{\prime} m\right\rangle$ vanishes unless $l^{\prime}=l \pm 1$, while the radial factor vanishes unless $l^{\prime}=l$.

In contrast to the energy correction, the first-order correction to the wavefunction has contribution from the dipole term only. Therefore: ${ }^{80}$

$$
\psi_{n l m}^{(1)}=R_{n l}(r) \sum_{l^{\prime}=l \pm 1} C_{l^{\prime} l m} Y_{l^{\prime} m}(\theta, \varphi)
$$

where mixing coefficients $C_{l^{\prime} l m}$ are proportional to the dipole moment $\mu$ :

$$
C_{l^{\prime} l m}=\frac{2 \mu}{l^{\prime}\left(l^{\prime}+1\right)-l(l+1)}\left\langle Y_{l^{\prime} m}|\cos \theta| Y_{l m}\right\rangle
$$

The contribution from the first two terms is zero as operator $1 / r$ is diagonal in $n, l$ and $m$ quantum numbers. The perturbation leaves the radial part unchanged and mixes the zero-order $l$-states with adjacent $l \pm 1$ states in the angular part, resulting in the orbital hybridization, as summarized for the first four states in Table V.

TABLE V: Details of the first-order orbital hybridizations arising from the dipole term of the perturbing Coulomb potential

\begin{tabular}{cc|cc|c|c}
\hline$\psi_{n l m}^{(0)}$ & \multicolumn{2}{|c|}{$\psi_{n l m}^{(1)}$} & \multirow{2}{*}{$\psi_{n l m}^{(0)}+\psi_{n l m}^{(1)}$} & State \\
\hline$l$ & $m$ & $l$ & $m$ & \\
\hline 0 & 0 & 1 & 0 & $s+p \sigma$ & ${ }^{2} \Sigma$ \\
1 & \pm 1 & 2 & \pm 1 & $p \pi+d \pi$ & ${ }^{2} \Pi$ \\
1 & 0 & 2,0 & 0 & $p \sigma+s+d \sigma$ & ${ }^{2} \Sigma$ \\
2 & \pm 2 & 3 & \pm 2 & $d+f$ & ${ }^{2} \Delta$ \\
\hline
\end{tabular}

Combining zero- and first-order contributions, the binding energy of the unpaired electron can be approximated as a second-order polynomial function of charge $Z$ on the metal:

$$
E_{n}=-\frac{1}{2 n^{2}}\left(Z^{2}-2 Z+2 Q\right)
$$

As the energy levels of a hydrogen-like atom are degenerate with $l$ and $m$, and the perturbation $V$ does not lift this degeneracy in the first order, the excitation energy is given by the 
energy difference between states with different principle quantum numbers:

$$
E_{n m}=E_{m}-E_{n}=\frac{1}{2}\left(\frac{1}{n^{2}}-\frac{1}{m^{2}}\right)\left(Z^{2}-2 Z+2 Q\right)
$$

Therefore, within (long-range) monopole approximation of the perturbation $V$, Eq. (11), the EOM-CC excitation energies are expected to depend quadratically on $Z$, as per Eq. (16). As one can clearly see from Figure 6, in which the molecules are arranged in the order of increasing electron-withdrawing strength (and therefore increasing $Z$ ), the quadratic dependence is not achieved. Thus, additional effects must be taken into account in order to reproduce the excitation energy trends, especially for the transitions to higher electronic states, where excitation energies display maximum for $\mathrm{MF}$ and $\mathrm{MOH}$. These effects missing in a simple one-electron model can be described by quantum defect theory.

\section{Quantum Defect Theory Fits to EOM-CC Data}

In a multi-electron atom, the effect of short-range interactions between the electron and the atomic core can be incorporated invoking quantum defect $\delta_{l}$ that changes $n$ to a non-integer effective quantum number $n^{*}$, Eq. (17). Quantum defect accounts for the penetration of the Rydberg electron into the cation core and depends on the $l$ quantum number associated with the given state:

$$
n^{*}=n-\delta_{l}
$$

Quantum defect can be determined directly from the Rydberg formula, Eq. (7), given that the binding energy $E_{n l}$ is known either from the experiment or from ab initio calculations:

$$
\delta_{l}=n-Z \sqrt{-\frac{1}{2 E_{n l}}}
$$

where $Z=2$ for alkaline earth metals and $E_{n l}$ is assumed negative.

The binding energies of singly ionized alkaline earth metals can be obtained directly from the EOM-EA-CCSD calculations using the dication state as reference. The calculations show that the binding energies of the valence $n s,(n-1) d$ and $n p$ states in $\mathrm{Ca}^{+}, \mathrm{Sr}^{+}, \mathrm{Ba}^{+}$increase when going to heavier ions, with the exception of $(n-1) d$ state that shows an energy decrease going from $\mathrm{Sr}^{+}$to $\mathrm{Ba}^{+}$(Table $\mathrm{S} 6$ in SI). Using the EOM-CC values of $E_{n l}$, quantum defects are 
then determined from Eq. (18). Because the probability to find the Rydberg electron within the core decreases with $l$, quantum defect decreases with $l$ (Table S6 in SI). At the same time, going to heavier ions, core penetration increases, which is reflected by the increasing values of quantum defect.

Because the atomic charges in molecules are not physical observables, their values are not unique and depend on the method of their derivation. We employ two definitions of partial atomic charges: Mulliken's and from the natural population analyses. The charges are computed using the CCSD wavefunction of the reference cation. Then for each set of charges, fit the coefficients of the second-order polynomial by taking the binding energies from the EOM-CC calculations as the reference values:

$$
y=a\left(Z^{2}+b Z+c\right)
$$

By comparing the polynomial coefficients in Eq. (19) with those in Eq. (15) derived above, the coefficient $a$ can be related to the quantum defect via $a=1 / 2\left(n-\delta_{l}\right)^{2}$, and the coefficients $b$ and $c$ reflect the Coulomb interaction of the Rydberg electron with the ligand and the conservation of the total charge.

Both fits closely reproduce the quadratic dependence of the binding energies on the atomic charges, as shown for the natural charges in Figure 9, and for Mulliken's charges in Figure S4 in SI. The fitted coefficients for both sets of charges are collected in Tables S8 and S7 in SI. They capture the overall physics of the developed model well. In particular, quantum defects $\delta_{l}$ obtained from the coefficient $a$ follow same trend as the quantum defects obtained for each ion: $\delta_{l}$ decreases with an increasing $l$ and increases for heavier ions. Furthermore, in both cases, the coefficients $b$ and $c$ have opposite signs, are similar in their magnitudes and close to the expected value of 2. Since the coefficient $c$ reflects the Coulomb interaction of the Rydberg electron with the charge of the molecular core, its slightly overestimated value suggests that the fitting procedure partially accommodates additional short-range interactions to correct the failing monopole approximation.

In order to separate (long-range) electrostatic interactions from (short-range) corepenetrating interactions, we fix the values of $b$ and $c$ to the values suggested by the model (that is, $-b=c=2$ ) and only adjust the quantum defect parameter to exactly reproduce the 
EOM-CC binding energies:

$$
\delta_{l}=n-\sqrt{-\frac{Z^{2}-2 Z+2 Q}{2 E_{n l}}}
$$

where $Q=1$ and $Z$ is the Mulliken or natural charge of the metal. Note that Eq. (20) is a modified version of Eq. (18), which accounts for the presence of the charged ligand.

The quantum defects are shown graphically in Figures 10 and S5 (in SI) for the natural and Mulliken's charges, respectively; numerical values are given in Tables S9 and S10 in SI. The data shows that quantum defects have an appreciable dependence on the ligand. While in the ground state this dependence is less prominent, in the excited states the plots show a clear lowering of the $\delta_{l}$ values for $\mathrm{MOH}$ and MF molecules, suggesting that the core-penetrating effects are the lowest for these molecules. The lower value of the quantum defect implies that the electronic structure of the molecule is closer to the electronic structure of an isolated ion, thus explaining the deviations from the overall trend in the excitation energies (Figure 6) and low magnitude of the bond length changes (Figure 7) for $\mathrm{MOH}$ and $\mathrm{MF}$.

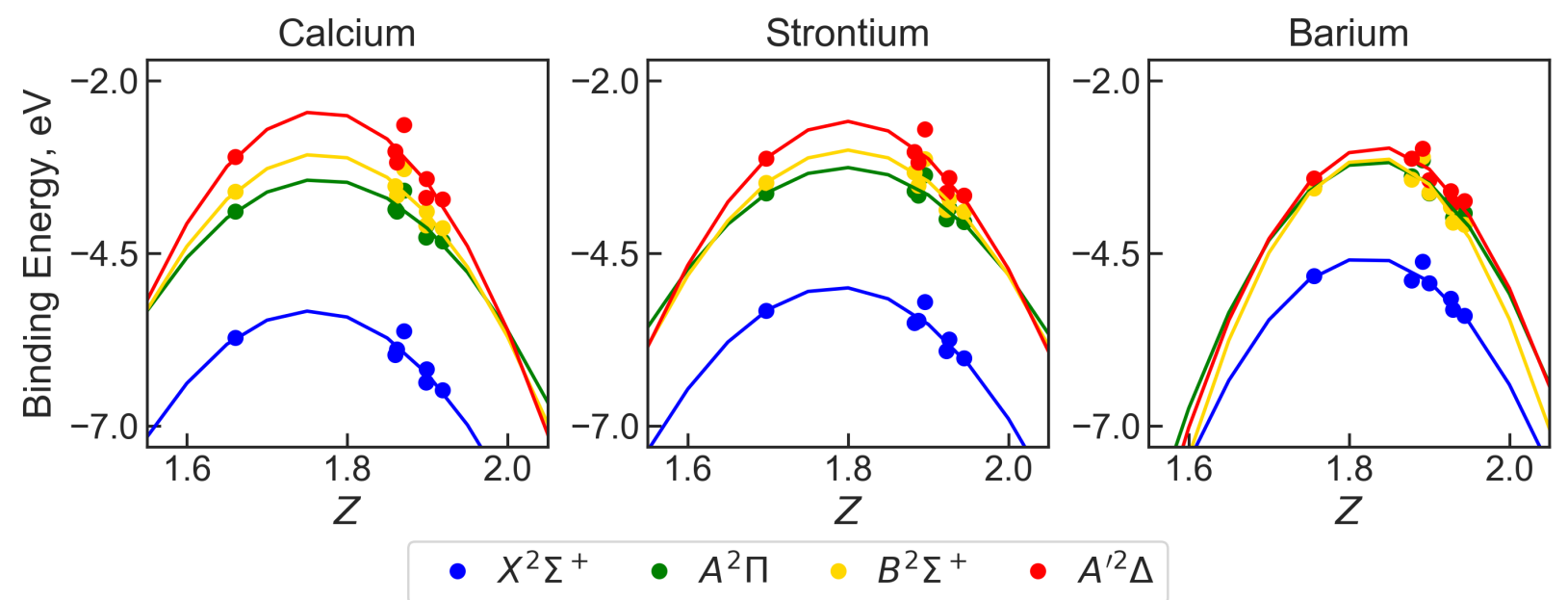

FIG. 9: Electron binding energies obtained using the EOM-EA-CCSD energies and natural charges. 


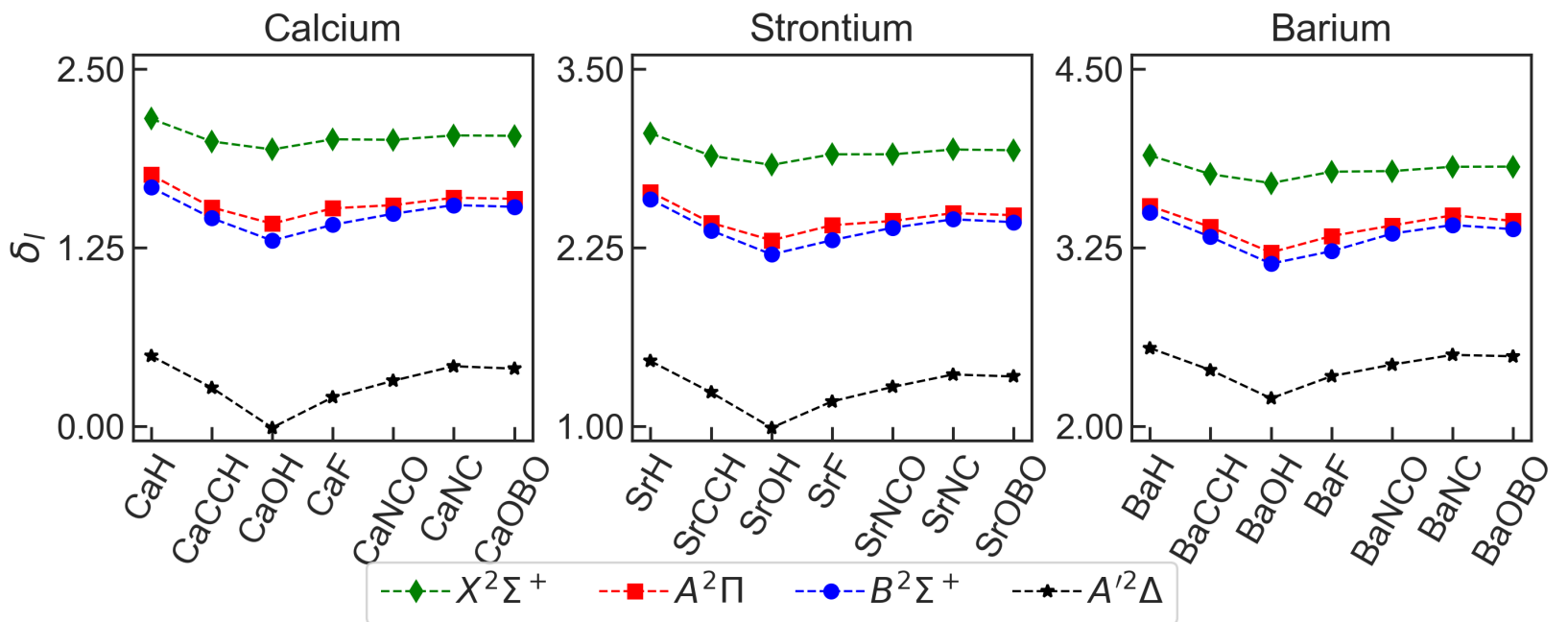

FIG. 10: Quantum defects $\delta_{l}$ in the MR series. 


\section{DISCUSSION}

In this computational study we investigated the electronic structure of monovalent alkaline earth metal derivatives $\mathrm{MR}$ in the context of their potential application in laser cooling. We characterized their optical and structural properties by systematically varying the cycling center and electron-withdrawing strength of the attached ligand using EOM-CC. We rationalized the computed properties using the effective Hamiltonian model.

In the MR molecules, one of the two valence electrons of the metal is transferred to the ligand. Consequently, the electronic structure of MR resembles that of the corresponding ionized alkaline earth metal $\mathrm{M}^{+}$. We visualized the extent of the locality of electron distribution and excitation by means of one-electron quantities such as Dyson orbitals and NTOs.

Dyson orbitals showed that the remaining unpaired electron is localized on the metal in the ground and the lowest three excited states, occupying hybridized atomic $s, p$ or $d$ orbitals. NTOs showed that upon electronic excitation, the change in the electron density can be represented by a single pair of hole and particle orbitals that are similar to the Dyson orbitals of the respective electronic state. Thus, the excitation does not extend beyond the cycling center, leading to nearly parallel PESs of the ground and excited states and, therefore, diagonal FCFs in this class of species.

While the metal and the ligand are ionically bound regardless of their type, the strength of the ionic bond and, therefore, the partial charge of the metal increases with the electronwithdrawing strength of the ligand. Our data showed that the excitation energies increase with the increasing electron-withdrawing strength due to the increasing long-range electrostatic interaction of the unpaired (Rydberg) electron with more positively charged metal. At the same time, when changing the cycling center to a heavier metal, the short-range core-penetrating interactions (manifested in the quantum defect $\delta_{l}$ ) increase, leading to the decrease in the excitation energies. Importantly, the quantum defect shows a clear dependence on the type of ligand in molecules with the same metal, with the lowest $\delta_{l}$ values corresponding to the metals attached to $\mathrm{F}$ and $\mathrm{OH}$.

Similarly, structural changes also depend on the interplay between the charge of the metal and its quantum defect. EOM-CC calculations showed that in CaR and SrR the magnitude

of the M-X bond length change generally decreases with the increasing electron-withdrawing strength of the ligand. Therefore, we anticipate more diagonal FCFs for the species such as 
$\operatorname{MOBO}(\mathrm{M}=\mathrm{Ca}$ and $\mathrm{Sr})$, where the OBO ligand has the largest known electron affinity. In the case of BaR, calculations showed that such molecules as $\mathrm{BaOBO}$ and $\mathrm{BaNC}$ have the largest bond length changes, suggesting that their FCFs are less diagonal. Interestingly, in all three series of alkaline earth metal derivatives, MF and $\mathrm{MOH}$ have the smallest M-X bond length changes despite having the ligand with moderate electron-withdrawing strength. This observation correlates with the smallest quantum defect values that have been determined for the metals attached to $\mathrm{F}$ and $\mathrm{OH}$.

\section{CONCLUSIONS}

On the basis of the above results, we conclude that within the chemical space of the alkaline earth metal derivatives, the most optimal candidate for laser cooling (that is, with the most diagonal FCFs) is expected to have an alkaline earth metal attached to such a ligand that reduces its quantum defect. Our data shows that metals attached to $\mathrm{R}=\mathrm{F}$ and $\mathrm{OH}$ have the smallest quantum defects, suggesting that $\mathrm{MF}$ and $\mathrm{MOH}$ are among the most suitable for laser-cooling. As $\mathrm{CaF}, \mathrm{SrF}$ and $\mathrm{SrOH}$ have been already laser-cooled, it is expected that $\mathrm{CaOH}$, $\mathrm{BaF}$ and $\mathrm{BaOH}$ are within a reach to be laser-cooled next, although presence of the low-lying $A^{\prime 2} \Delta$ state in barium derivatives may provide additional decay paths that should be addressed. Furthermore, calcium and strontium derivatives may benefit from the ligands that withdraw as much electron density as possible from the cycling center, suggesting that such molecules as MOBO and MNC ( $\mathrm{M}=\mathrm{Ca}$ and $\mathrm{Sr})$ could be promising candidates for laser cooling.

Clearly, a set of possible ligands that could be attached to the cycling center is not limited to those considered in this work and other more complex candidates are possible, including polyatomic inorganic and aromatic superhalogens. Future studies are necessary to validate whether the design principle proposed here is also applicable to other ligands and metals including rare earth, transition, main group metals and lanthanides.

\section{ACKNOWLEDGMENT}

This work was supported by the "Molecules Functionalized with Optical Cycling Centers" collaboration, which is supported by the U.S. Department of Energy (Award de-sc0019245). A.I.K. is also a grateful recipient of the Simons Fellowship in Theoretical Physics and of the 
Mildred Dresselhaus Award, which supported her sabbatical stay in Germany.

Supporting information is available: Detailed information on the results from EOM-CC calculations (Dyson orbitals, equilibrium bond lengths, excitation energies, oscillator strengths), their comparison with available experimental data and quantum defects data. 
1 derStraten, P. Van; Metcalf, H. Atoms and molecules interacting with light: Atomic physics for the laser era; Cambridge University Press, 2016.

2 Quemener, G.; Julienne, P. S. Ultracold molecules under control! Chem. Rev. 2012, 112, 49495011.

3 Hudson, E. R.; Campbell, W. C. Dipolar quantum logic for freely rotating trapped molecular ions Phys. Rev. A 2018, 98, 040302.

4 Ni, K. K.; Rosenband, T.; Grimes, D. D. Dipolar exchange quantum logic gate with polar molecules Chem. Sci. 2018, 9, 6830-6838.

5 Yang, T.; Li, A.; Chen, G. K.; Xie, C.; Suits, A. G.; Campbell, W. C.; Guo, H.; Hudson, E. R. Optical control of reactions between water and laser-cooled $\mathrm{Be}^{+}$ions J. Phys. Chem. Lett. 2018, 9, 3555-3560.

6 Hutson, J. M. Ultracold chemistry Science 2010, 327, 788-789.

7 Bell, M. T.; Softley, T. P. Ultracold molecules and ultracold chemistry Mol. Phys. 2009, 10\%, 99-132.

8 Kozyryev, I.; Hutzler, N. R. Precision measurement of time-reversal symmetry violation with lasercooled polyatomic molecules Phys. Rev. Lett. 2017, 119, 133002.

9 Rosa, M. D. Di Laser-cooling molecules Eur. Phys. J. D 2004, 31, 395-402.

10 Isaev, T. A.; Hoekstra, S.; Berger, R. Laser-cooled RaF as a promising candidate to measure molecular parity violation Phys. Rev. A 2010, 82, 052521.

11 Wells, N.; Lane, I. C. Electronic states and spin-forbidden cooling transitions of AlH and AlF Phys. Chem. Chem. Phys. 2011, 13, 19018-19025.

12 Nguyen, J. H. V.; Viteri, C. R.; Hohenstein, E. G.; Sherrill, C. D.; Brown, K. R.; Odom, B. Challenges of laser-cooling molecular ions New J. Phys. 2011, 13, 063023.

13 Gao, Y.; Gao, T. Laser cooling of the alkaline-earth-metal monohydrides: Insights from an ab initio theory study Phys. Rev. A 2014, 90, 052506.

14 Wan, M.-J.; Shao, J.-X.; Huang, D.-H.; Jin, C.-G.; Yu, Y.; Wang, F.-H. Laser cooling of BeCl and BeBr molecules in an ab initio method Phys. Chem. Chem. Phys. 2015, 17, 26731-26739.

15 Li, C.; Li, Y.; Ji, Z.; Qiu, X.; Lai, Y.; Wei, J.; Zhao, Y.; Deng, L.; Chen, Y.; Liu, J. Candidates for direct laser cooling of diatomic molecules with the simplest ${ }^{1} \Sigma-{ }^{1} \Sigma$ electronic system Phys. Rev. 
A 2018, 97, 062501.

Isaev, T. A.; Zaitsevskii, A. V.; Eliav, E. Laser-coolable polyatomic molecules with heavy nuclei $J$. Phys. B 2017, 50, 225101.

17 Gaul, K.; Berger, R. Ab initio study of parity and time-reversal violation in laser-coolable triatomic molecules arXiv 2018; in press; preprint: https://arxiv.org/abs/1811.05749.

18 Li, M.; Kłos, J.; Petrov, A.; Kotochigova, S. Emulating optical cycling centers in polyatomic molecules arXiv 2019; in press; preprint: https://arxiv.org/abs/1904.11579.

19 O'Rourke, M. J.; Hutzler, N. R. Hypermetallic polar molecules for precision measurements arXiv 2019; in press; preprint: https://arxiv.org/abs/1902.10683.

20 Shuman, E. S.; Barry, J. F.; DeMille, D. Laser cooling of a diatomic molecule Nature 2010, 467, 820.

21 Barry, JF; McCarron, DJ; Norrgard, EB; Steinecker, MH; DeMille, D Magneto-optical trapping of a diatomic molecule Nature 2014, 512, 286.

22 Hummon, M. T.; Yeo, M.; Stuhl, B. K.; Collopy, A. L.; Xia, Y.; Ye, J. 2D magneto-optical trapping of diatomic molecules Phys. Rev. Lett. 2013, 110, 143001.

23 Collopy, A. L.; Ding, S.; Wu, Y.; Finneran, I. A.; Anderegg, L.; Augenbraun, B. L.; Doyle, J. M.; Ye, J. 3D magneto-optical trap of yttrium monoxide Phys. Rev. Lett. 2018, 121, 213201.

24 Zhelyazkova, V.; Cournol, A.; Wall, T. E.; Matsushima, A.; Hudson, J. J.; Hinds, E. A.; Tarbutt, M. R.; Sauer, B. E. Laser cooling and slowing of CaF molecules Phys. Rev. A 2014, 89, 053416.

25 Anderegg, L.; Augenbraun, B. L.; Bao, Y.; Burchesky, S.; Cheuk, L. W.; Ketterle, W.; Doyle, J. M. Laser cooling of optically trapped molecules Nat. Phys. 2018, 14, 890-893.

26 Truppe, S; Williams, HJ; Hambach, M; Caldwell, L; Fitch, NJ; Hinds, EA; Sauer, BE; Tarbutt, MR Molecules cooled below the Doppler limit Nat. Phys. 2017, 13, 1173.

27 Lim, J.; Almond, J. R.; Trigatzis, M. A.; Devlin, J. A.; Fitch, N. J.; Sauer, B. E.; Tarbutt, M. R.; Hinds, E. A. Laser cooled YbF molecules for measuring the electron's electric dipole moment Phys. Rev. Lett. 2018, 120, 123201.

28 Kozyryevn, I.; Baum, L.; Matsuda, K.; Augenbraun, B. L.; Anderegg, L.; Sedlack, A. P.; Doyle, J. M. Sisyphus laser cooling of a polyatomic molecule Phys. Rev. Lett. 2017, 118, 173201.

29 Isaev, T. A.; Berger, R. Polyatomic candidates for cooling of molecules with lasers from simple theoretical concepts Phys. Rev. Lett. 2016, 116, 063006.

30 Kozyryev, I.; Baum, L.; Matsuda, K.; Doyle, J. M. Proposal for laser cooling of complex polyatomic 
molecules ChemPhysChem 2016, 17, 3641-3648.

31 Krylov, A. I. Equation-of-motion coupled-cluster methods for open-shell and electronically excited species: The hitchhiker's guide to Fock space Annu. Rev. Phys. Chem. 2008, 59, 433-462.

Sneskov, K.; Christiansen, O. Excited state coupled cluster methods WIREs: Comput. Mol. Sci. 2012, 2, 566-584.

Bartlett, R. J. Coupled-cluster theory and its equation-of-motion extensions WIREs: Comput. Mol. Sci. 2012, 2, 126-138.

34 Rice, S. F.; Martin, H.; Field, R. W. The electronic structure of the calcium monohalides. A ligand field approach J. Chem. Phys. 1985, 82, 5023-5034.

35 Törring, T.; Ernst, W. E.; Kändler, J. Energies and electric dipole moments of the low lying electronic states of the alkaline earth monohalides from an electrostatic polarization model $J$. Chem. Phys. 1989, 90, 4927-4932.

36 Harris, N. A.; Jungen, C. Rydberg states of calcium fluoride Phys. Rev. Lett. 1993, 70, 2549.

37 Jungen, C. Molecular applications of quantum defect theory; CRC Press, 1996.

38 Li, W.; Mourachko, I.; Noel, M. W.; Gallagher, T. F. Millimeter-wave spectroscopy of cold Rb Rydberg atoms in a magneto-optical trap: Quantum defects of the $n s, n p$, and nd series Phys. Rev. A 2003, 67, 052502.

39 Kay, J. J.; Coy, S. L.; Wong, B. M.; Jungen, C.; Field, R. W. A quantum defect model for the $s$, $p, d$, and $f$ Rydberg series of CaF J. Chem. Phys. 2011, 134, 114313.

40 Li, J.; Liu, Y.; Moss, D. B.; Gittins, C. M.; Harris, N. A.; Field, R. W. Double-resonance spectroscopic studies of core-penetrating Rydberg states of CaCl J. Molec. Spect. 1999, 193, 403-411.

41 Arif, M.; Jungen, C.; Roche, A. L. The Rydberg spectrum of CaF and BaF: Calculation by Rmatrix and generalized quantum defect theory J. Chem. Phys. 1997, 106, 4102-4118.

42 Jakubek, Z. J.; Field, R. W. Core-penetrating Rydberg series of BaF: $s \sim p \sim d \sim f$ supercomplexes Phys. Rev. Lett. 1994, 72, 2167.

43 Reisler, H.; Krylov, A. I. Interacting Rydberg and valence states in radicals and molecules: Experimental and theoretical studies Int. Rev. Phys. Chem. 2009, 28, 267-308.

44 Samanta, B.; Sutradhar, S.; Fernando, R.; Krylov, A. I.; Reisler, H. Electronic structure and Rydberg-core interactions in hydroxycarbene and methylhydroxycarbene J. Phys. Chem. A 2018, 122, 6176-6182.

45 Krylov, A. I. In Reviews in Comp. Chem.; Parrill, A.L., Lipkowitz, K.B., Eds., Vol. 30; J. Wiley 
\& Sons, 2017; p. 151.

46 Nijamudheen, A.; Akimov, A. V. Criticality of symmetry in rational design of chalcogenide perovskites J. Phys. Chem. Lett. 2017, 9, 248-257.

47 Ivanov, M. V.; Reid, S. A.; Rathore, R. Game of frontier orbitals: a view on the rational design of novel charge-transfer materials J. Phys. Chem. Lett. 2018, 9, 3978-3986.

Linderberg, J.; Öhrn, Y. Propagators in quantum chemistry; Academic, London, 1973.

49 Ortiz, J.V. Toward an exact one-electron picture of chemical bonding Adv. Quantum Chem. 1999, $35,33-52$.

50 Oana, C. M.; Krylov, A. I. Dyson orbitals for ionization from the ground and electronically excited states within equation-of-motion coupled-cluster formalism: Theory, implementation, and examples J. Chem. Phys. 2007, 127, 234106-14.

51 Luzanov, A.V.; Sukhorukov, A.A.; Umanskii, V.E. Application of transition density matrix for analysis of excited states Theor. Exp. Chem. 1976, 10, 354-361.

52 Head-Gordon, M.; Grana, A. M.; Maurice, D.; White, C. A. Analysis of electronic transitions as the difference of electron attachment and detachment densities J. Phys. Chem. 1995, 99, 14261 14270.

53 Martin, R.L. Natural transition orbitals J. Phys. Chem. A 2003, 118, 4775-4777.

54 Luzanov, A.V.; Zhikol, O.A. In Practical aspects of computational chemistry I: An overview of the last two decades and current trends; Leszczynski, J., Shukla, M.K., Eds.; Springer, 2012; p. 415.

55 Plasser, F.; Wormit, M.; Dreuw, A. New tools for the systematic analysis and visualization of electronic excitations. I. Formalism J. Chem. Phys. 2014, 141, 024106-13.

56 Plasser, F.; Bäppler, S.A.; Wormit, M.; Dreuw, A. New tools for the systematic analysis and visualization of electronic excitations. II. Applications J. Chem. Phys. 2014, 141, 024107-12.

57 Mewes, S.; Plasser, F.; Krylov, A. I.; Dreuw, A. Benchmarking excited-state calculations using exciton properties J. Chem. Theory Comput. 2018, 14, 710-725.

58 Oana, C. M.; Krylov, A. I. Cross sections and photoelectron angular distributions in photodetachment from negative ions using equation-of-motion coupled-cluster Dyson orbitals J. Chem. Phys. 2009, 131, 124114-15.

59 Y. Shao, Z. Gan, E. Epifanovsky, A.T.B. Gilbert, M. Wormit, J. Kussmann, A.W. Lange, A. Behn, J. Deng, X. Feng, D. Ghosh, M. Goldey, P.R. Horn, L.D. Jacobson, I. Kaliman, R.Z. Khaliullin, T. Kus, A. Landau, J. Liu, E.I. Proynov, Y.M. Rhee, R.M. Richard, M.A. Rohrdanz, R.P. Steele, 
E.J. Sundstrom, H.L. Woodcock III, P.M. Zimmerman, D. Zuev, B. Albrecht, E. Alguires, B. Austin, G.J.O. Beran, Y.A. Bernard, E. Berquist, K. Brandhorst, K.B. Bravaya, S.T. Brown, D. Casanova, C.-M. Chang, Y. Chen, S.H. Chien, K.D. Closser, D.L. Crittenden, M. Diedenhofen, R.A. DiStasio Jr., H. Do, A.D. Dutoi, R.G. Edgar, S. Fatehi, L. Fusti-Molnar, A. Ghysels, A. Golubeva-Zadorozhnaya, J. Gomes, M.W.D. Hanson-Heine, P.H.P. Harbach, A.W. Hauser, E.G. Hohenstein, Z.C. Holden, T.-C. Jagau, H. Ji, B. Kaduk, K. Khistyaev, J. Kim, J. Kim, R.A. King, P. Klunzinger, D. Kosenkov, T. Kowalczyk, C.M. Krauter, K.U. Laog, A. Laurent, K.V. Lawler, S.V. Levchenko, C.Y. Lin, F. Liu, E. Livshits, R.C. Lochan, A. Luenser, P. Manohar, S.F. Manzer, S.-P. Mao, N. Mardirossian, A.V. Marenich, S.A. Maurer, N.J. Mayhall, C.M. Oana, R. OlivaresAmaya, D.P. O’Neill, J.A. Parkhill, T.M. Perrine, R. Peverati, P.A. Pieniazek, A. Prociuk, D.R. Rehn, E. Rosta, N.J. Russ, N. Sergueev, S.M. Sharada, S. Sharmaa, D.W. Small, A. Sodt, T. Stein, D. Stuck, Y.-C. Su, A.J.W. Thom, T. Tsuchimochi, L. Vogt, O. Vydrov, T. Wang, M.A. Watson, J. Wenzel, A. White, C.F. Williams, V. Vanovschi, S. Yeganeh, S.R. Yost, Z.-Q. You, I.Y. Zhang, X. Zhang, Y. Zhou, B.R. Brooks, G.K.L. Chan, D.M. Chipman, C.J. Cramer, W.A. Goddard III, M.S. Gordon, W.J. Hehre, A. Klamt, H.F. Schaefer III, M.W. Schmidt, C.D. Sherrill, D.G. Truhlar, A. Warshel, X. Xu, A. Aspuru-Guzik, R. Baer, A.T. Bell, N.A. Besley, J.-D. Chai, A. Dreuw, B.D. Dunietz, T.R. Furlani, S.R. Gwaltney, C.-P. Hsu, Y. Jung, J. Kong, D.S. Lambrecht, W.Z. Liang, C. Ochsenfeld, V.A. Rassolov, L.V. Slipchenko, J.E. Subotnik, T. Van Voorhis, J.M. Herbert, A.I. Krylov, P.M.W. Gill, and M. Head-Gordon Advances in molecular quantum chemistry contained in the Q-Chem 4 program package Mol. Phys. 2015, 113, 184-215.

60 Krylov, A. I.; Gill, P. M. W. Q-Chem: An engine for innovation WIREs: Comput. Mol. Sci. 2013, 3, 317-326.

61 Müller, W.; Flesch, J.; Meyer, W. Treatment of intershell correlation effects in ab initio calculations by use of core polarization potentials. method and application to alkali and alkaline earth atoms J. Chem. Phys. 1984, 80, 3297-3310.

62 Iron, M. A.; Oren, M.; Martin, J. M. L. Alkali and alkaline earth metal compounds: core - valence basis sets and importance of subvalence correlation Mol. Phys. 2003, 101, 1345-1361.

63 Hill, J. G.; Peterson, K. A. Gaussian basis sets for use in correlated molecular calculations. XI. pseudopotential-based and all-electron relativistic basis sets for alkali metal (K-Fr) and alkaline earth (Ca-Ra) elements J. Chem. Phys. 2017, 147, 244106.

64 Lim, I. S.; Schwerdtfeger, P.; Metz, B.; Stoll, H. All-electron and relativistic pseudopotential studies 
for the group 1 element polarizabilities from K to element 119 J. Chem. Phys. 2005, 122, 104103.

Lim, I. S.; Stoll, H.; Schwerdtfeger, P. Relativistic small-core energy-consistent pseudopotentials for the alkaline-earth elements from Ca to Ra J. Chem. Phys. 2006, 124, 034107.

Dunning, Jr., T. H. Gaussian basis sets for use in correlated molecular calculations. I. The atoms boron through neon and hydrogen J. Chem. Phys. 1989, 90, 1007-1023.

67 Mulliken, R.S. Electronic population analysis on LCAO-MO molecular wave functions. I J. Chem. Phys. 1955, 23, 1833-1840.

68 Weinhold, F.; Landis, C.R. Discovering Chemistry With Natural Bond Orbitals; New Jersey: John Wiley \& Sons, 2012.

69 ezSpectrum. Mozhayskiy, V. A.; Krylov, A. I.;

ezSpectrum, http://iopenshell.usc.edu/downloads/.

70 Brazier, C. R.; Ellingboe, L. C.; Kinsey-Nielsen, S.; Bernath, P. F. Laser spectroscopy of alkaline earth monoalkoxide free radicals J. Am. Chem. Soc. 1986, 108, 2126-2132.

71 Bernath, P. F. Spectroscopy and photochemistry of polyatomic alkaline earth containing molecules Adv. Photochem. 1997, 23, 1-62.

72 Ellis, A. M. Main group metal-ligand interactions in small molecules: New insights from laser spectroscopy Int. Rev. Phys. Chem. 2001, 20, 551-590.

73 Koput, J.; Peterson, K. A. Ab Initio potential energy surface and vibrational-rotational energy levels of $X^{2} \Sigma^{+} \mathrm{CaOH}$ J. Phys. Chem. A 2002, 106, 9595-9599.

74 Hao, Y.; Pašteka, L. F.; Visscher, L.; Aggarwal; the NL-eEDM collaboration:P. Aggarwal; Bethlem, H. L.; Boeschoten, A.; Borschevsky, A.; Denis, M.; Esajas, K.; Hoekstra, S.; Jungmann, K.; Marshall, V. R.; Meijknecht, T. B.; Mooij, M. C.; Timmermans, R. G. E.; Touwen, A.; Ubachs, W.; Willmann, L.; Yin, Y.; Zapara, A. High accuracy theoretical investigations of $\mathrm{CaF}, \mathrm{SrF}$, and $\mathrm{BaF}$ and implications for laser-cooling arXiv 2019; in press; preprint: https://arxiv.org/abs/1904.02516.

75 Bernath, P. F. Gas-phase inorganic chemistry: monovalent derivatives of calcium and strontium Science 1991, 254, 665-670.

76 Gutsev, G. L.; Boldyrev, A. I. DVM-X $\alpha$ calculations on the ionization potentials of $\mathrm{MX}_{k+1}^{-}$complex anions and the electron affinities of $\mathrm{MX}_{k+1}$ "superhalogens" Chem. Phys. 1981, 56, 277-283.

77 Zhai, H.-J.; Wang, L.-M.; Li, S.-D.; Wang, L.-S. Vibrationally resolved photoelectron spectroscopy of $\mathrm{BO}^{-}$and $\mathrm{BO}_{2}^{-}$: A joint experimental and theoretical study J. Phys. Chem. A 2007, 111, 
$1030-1035$.

78 Kozyryev, I.; Steimle, T. C.; Yu, P.; Nguyen, D.-T.; Doyle, J. M. Determination of CaOH and $\mathrm{CaOCH}_{3}$ vibrational branching ratios for direct laser cooling and trapping New J. Phys. 2019, 21, 052002.

79 Nguyen, D.-T.; Steimle, T. C.; Kozyryev, I.; Huang, M.; McCoy, A. B. Fluorescence branching ratios and magnetic tuning of the visible spectrum of $\mathrm{SrOH}$ J. Molec. Spect. 2018, 347, 7-18.

80 Watson, J. K. G. Effects of a core electric dipole moment on Rydberg states Mol. Phys. 1994, 81, $277-289$. 
TOC graphics

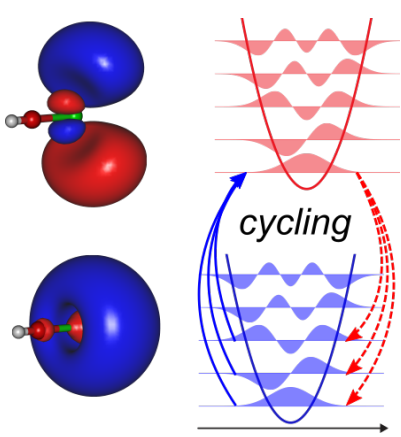

